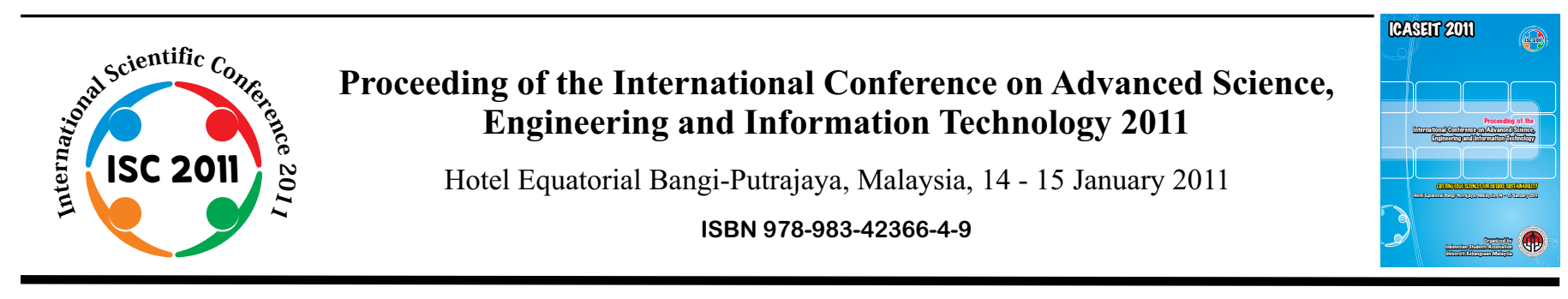

\title{
A Temperature Total Fourier Series Solution For a Hollow Sphere
}

\author{
Mehdi Mahmudi Mehrizi \\ Department of Mechanical Engineering ,Islamic Azad University ,Mehriz Branch \\ Address:Mehriz,Iran \\ Tel:+983525229100,E-mail: mehdi.mahmodi@gmail.com
}

\begin{abstract}
In the following pages, we exhibit an analytical solution of a two-dimensional temperature field in a hollow sphere under total periodic boundary condition. The material is assumed to be homogeneous and isotropic with time-independent thermal properties .Till now periodic boundary condition was derived with a harmonic vibration, whereas there is a noticeable difference in the practical conditions with harmonic vibration .In this essay, by means of Fourier analysis, we imagine the outside total periodic boundary condition, as aggregate of harmonic vibrations. To solve the problem, first we imagine the boundary condition as constant values and with separation of variables; we can obtain temperature distribution in the sphere. Then Duhamel's theorem is used to calculate temperature field under fully periodic boundary condition. For confirmation of accurate solution, we can compare the result for a harmonic vibration and those reported by others. Also, solutions for a hollow sphere were compared with other present references. At last we can obtain thermal stresses which is caused by temperature field in the hollow sphere
\end{abstract}

Keywords-Hollow Sphere, Fourier Series , conduction

\section{INTRODUCTION}

There are several heat conduction problems that can be modeled by a sphere of constant properties, for example, food freezing and the hydrocooling of spherical fruits or vegetables [1]. The solution of some cases of heat conduction problems can be found in heat transfer literature. Heat conduction problems with periodic boundary condition have some applications in engineering such as

penetration of the daily and annual temperature cycles into the earth's surface, heating up and cooling down phases in the Siemens Martin glass melting furnaces, wall temperature oscillation of internal combustion engines, experimental methods for specifying the thermal diffusivity of materials [2] and the temperature field [3,4], and also the thermal stresses caused by temperature distribution. Trostel calculated thermal stresses caused by thermal loads in a solid sphere [5]. Zubair and Chaudhry discussed the solution for temperature and heat flux in a semi-infinite solid subject to periodic-type surface heat fluxes [6]. The calculation of temperature distribution in a solid sphere under a periodic boundary condition is presented in [4] that is simulated by harmonic

oscillation of the ambient temperature. The purpose of this paper is to derive an analytical solution for a two- dimensional heat conduction in a hollow sphere, subjected to a periodic boundary condition. As for the validity of the results,

a comparison between the temperature distribution in a solid sphere with the theoretical ones [4] is presented for the same boundary condition. The results can be used for approximation to the real problems with periodic boundary condition. They can also be utilized to verify the time consuming complex computer calculations.

\section{MATHEMATICAL MODEL}

The heat conduction equation in spherical coordinates for an isotropic material that has temperature and timeindependent properties, and without heat source under axisymmetric condition, is ,:

$a^{2} \frac{\partial \theta}{\partial t}=\frac{\partial^{2} \theta}{\partial r^{2}}+\frac{2}{r} \frac{\partial \theta}{\partial r}+\frac{1}{r^{2}}\left(\cot \psi \frac{\partial \theta}{\partial \psi}+\frac{\partial^{2} \theta}{\partial \psi^{2}}\right)$

The initial temperature of the ambient and the hollow sphere are zero. The inner boundary condition is insulated and the outer one is assumed to be boundary condition of type 3 : 


$$
\left\{\begin{array}{l}
\theta\left(r_{o}, \psi, t\right)+\left.\frac{k}{h} \frac{\partial \theta}{\partial r}\right|_{r_{o}, \psi, t}=\Theta_{M}(\psi, t) \\
\left.\frac{\partial \theta}{\partial r}\right|_{r_{i}, \psi, t}=0
\end{array}\right.
$$

We consider that $\Theta_{M}(\psi, t)=g(t) f_{o}(\psi)$ where $g(t)$ is assumed to be a periodic function that is decomposed using Fourier series:

$g(t)=\bar{\Theta}+\sum_{m=0}^{\infty} \Theta_{m} \sin \left(2 m \pi \frac{t}{p}\right)+\sum_{m=0}^{\infty} \bar{\Theta}_{m} \cos \left(2 m \pi \frac{t}{p}\right)$

and $f_{o}(\psi)$ is an arbitrary function.

An easy way to comply with the conference paper formatting requirements is to use this document as a template and simply type your text into it.

and $f_{o}(\psi)$ is an arbitrary function.

\section{A. Analytical Solution}

The problem cannot be solved directly because of the dependency of nonhomogeneous term, $\Theta_{M}(\psi, t)$, on time [5]. The solution of a heat-conduction problem with timedependent boundary condition can be related to the solution of the same problem with time-independent boundary condition by means of Duhamel's theorem. Thus, first of all, the equation should be solved with the

assumption that the boundary condition is time independent. In this situation the boundary and initial conditions are:

$\left\{\begin{array}{l}\theta\left(r_{o}, \psi, t\right)+\left.\frac{k}{h} \frac{\partial \theta}{\partial r}\right|_{r_{o}, \psi, t}=f_{o}(\psi) \\ \left.\frac{\partial \theta}{\partial r}\right|_{r_{i}, \psi, t}=0\end{array}\right.$

$$
\theta(r, \psi, 0)=0
$$

In this case, use was made of the superposition principle; the solution is the sum of a steady solution, $\theta_{0}(r, \psi)$, and a transient solution $\theta_{1}(r, \psi, t)$. The differential heat conduction equation in steady form is:

$$
\frac{\partial^{2} \theta_{0}}{\partial r^{2}}+\frac{2}{r} \frac{\partial \theta_{0}}{\partial r}+\frac{1}{r^{2}}\left(\cot \psi \frac{\partial \theta_{0}}{\partial \psi}+\frac{\partial^{2} \theta_{0}}{\partial \psi^{2}}\right)=0
$$

where the boundary condition is given by Eq. (4). The transient differential equation is:

$$
a^{2} \frac{\partial \theta_{1}}{\partial t}=\frac{\partial^{2} \theta_{1}}{\partial r^{2}}+\frac{2}{r} \frac{\partial \theta_{1}}{\partial r}+\frac{1}{r^{2}}\left(\cot \psi \frac{\partial \theta_{1}}{\partial \psi}+\frac{\partial^{2} \theta_{1}}{\partial \psi^{2}}\right)
$$

and the following conditions must be satisfied

$$
\begin{aligned}
& \left\{\begin{array}{l}
\theta_{1}\left(r_{o}, \psi, t\right)+\left.\frac{k}{h} \frac{\partial \theta_{1}}{\partial r}\right|_{r_{o}, \psi, t}=0 \\
\left.\frac{\partial \theta_{1}}{\partial r}\right|_{r_{i}, \psi, t}=0
\end{array}\right. \\
& \theta_{1}(r, \psi, 0)=-\theta_{0}(r, \psi)
\end{aligned}
$$

\section{B. Steady-State Problem.}

To solve Eq. (6), the method of separation of variables is used. Two differential equations are obtained, a Euler type and a Legendre type. Then, by applying Eq. (4), the solution of the steady state is:

$$
\theta_{0}(r, \zeta)=\sum_{n=0}^{\aleph} C_{n} \eta_{n}(r) P_{n}(\zeta) \quad \zeta(\psi)=\cos (\psi)
$$

Where:

$$
\eta_{n}(r)=\frac{-(n+1) r_{i}^{-(n+2)}}{\Delta^{(n)}} r^{n}-\frac{-n r_{i}^{(n-1)}}{\Delta^{(n)}} r^{-(n+1)}
$$

$$
\begin{gathered}
\left\{\Delta^{(n)}=-(n+1) r_{i}^{-(n+2)}\left(r_{o}^{n}+n \frac{k}{h} r_{o}^{(n-1)}\right)-n r_{i}^{(n-1)}\left(r_{o}^{-(n+1)}-(n+1) \frac{k}{h} r_{o}^{-(n+2)}\right)\right. \\
C_{n}=\frac{2 n+1}{2} \int_{-1}^{+1} f_{o}(\varsigma) P_{n}(\varsigma) d \varsigma
\end{gathered}
$$

\section{Transient Problem}

Use was made of the method of separation of variables to solve Eq. (7). The boundary equation, Eq. (8), and Eq. (9) should be satisfied. When eigenvalues, $\omega_{k n}$ are calculated, the final solution of the transient problem can be expressed as:

$$
\theta_{1}(r, \psi, t)=-\sum_{k=0}^{\infty} \sum_{n=0}^{\infty} \frac{1}{\delta_{k n}} \Phi_{n}\left(\omega_{k n} r\right) P_{n}(\zeta) e^{-\left(\frac{\omega_{k n}}{a}\right)^{2} t} \int_{r_{i}}^{r_{o}} r^{2} \eta_{n}(r) \Phi_{n}\left(\omega_{k n} r\right) d r
$$

Where 
$\Phi_{n}\left(\omega_{k n} r\right)=\frac{L_{k n}}{\sqrt{r}}\left\{\left[J_{-\left(n+\frac{1}{2}\right)}\left(\omega_{k n} r_{o}\right)+\frac{k}{2 h r_{0}}\left(2 \omega_{k n} r_{0}^{\prime} J_{-\left(n+\frac{1}{2}\right)}^{\prime}\left(\omega_{k n} r_{0}\right)-J_{-\left(n+\frac{1}{2}\right)}\left(\omega_{k n} r_{0}\right)\right)\right] J_{n+\frac{1}{2}}\left(\omega_{k n} r\right)\right.$

$-\left[J_{\left(n+\frac{1}{2}\right)}\left(\omega_{k n} r_{o}\right)+\frac{k}{2 h r_{0}}\left(2 \omega_{k n} r_{0} J_{\left(n+\frac{1}{2}\right)}^{\prime}\left(\omega_{k n} r_{0}\right)-J_{\left(n+\frac{1}{2}\right)}\left(\omega_{k n} r_{0}\right)\right) J_{-\left(n+\frac{1}{2}\right)}\left(\omega_{k n} r\right)\right.$

\section{Temperature Field Under Time Varying Boundary Condition.}

As we mentioned before, the temperature distribution under a constant boundary condition is the summation of steady and transient states:

$\theta(r, \psi, t)=\sum_{n=0}^{\infty}\left(\eta_{n}(r)-\sum_{k=0}^{\infty} \frac{1}{\delta_{k n}} e^{-\left(\frac{\omega_{k n}}{a}\right)^{2} t} \Phi_{n}\left(\omega_{k n} r\right) \int_{r_{i}}^{r_{o}} r^{2} \eta_{n}(r) \Phi_{n}\left(\omega_{k n} r\right) d r\right) P_{n}(\zeta)$

Equation (17) expresses the temperature field under time independent boundary condition. In the case, the boundary values depend on time; they have the variations in the forms:

$$
d C_{n}=\frac{d C_{n}}{d \tau} d \tau
$$

It can be assumed that $\frac{d C_{n}}{d \tau}$ are constant at some time, $\tau$ Therefore the temperature distribution after $t-\tau$ seconds after the beginning of the influences can be expressed in the form (7):

$d \theta(r, \psi, t)=\sum_{n=0}^{\infty}\left(\eta_{n}^{(o)}(r)-\sum_{k=0}^{\infty} \frac{1}{\delta_{k n}} e^{-\left(\frac{\omega_{n}}{a}\right)^{2}(t-\tau)} \Phi_{n}\left(\omega_{k n} r\right) \int_{r_{i}}^{r_{o}} r^{2} \eta_{n}^{(o)}(r) \Phi_{n}\left(\omega_{k n} r\right) d r\right) P_{n}(\zeta) \frac{d C_{n}}{d \tau} d \tau$

Thus, the temperature field can be obtained by summation of $d C_{n}$ during $d \tau$ and the influence of $C_{n}(0)=0$ The following equation is proven by the method of integration by parts:

$C_{n}(0) e^{-\left(\frac{\omega_{k n}}{a}\right)^{2} t}+\int_{\tau=0}^{t} e^{-\left(\frac{\omega_{\frac{k n}{n}}}{a}\right)^{2}(t-\tau)} \frac{d C_{n}}{d \tau} d \tau=C_{n}(t)-\left(\frac{\omega_{k n}}{a}\right)^{2} \int_{\tau=0}^{t} C_{n}(t) e^{-\left(\frac{\omega_{k n}}{a}\right)^{2}(t-\tau)} d \tau$

Making use of Eq. (20), the temperature field can be obtained in the simplified form:

$\theta(r, \psi, t)=\sum_{n=0}^{\infty} \sum_{k=0}^{\infty} D_{k n} \Phi_{n}\left(\omega_{k n} r\right) P_{n}(\zeta) T_{k n}(t)$

Where :

$D_{k n}=\frac{\omega_{k n}^{2}}{a^{2} \delta_{k n}}$

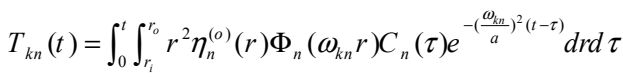

The heat flux of the outer surface of the sphere can be calculated by:
$Q=-\int_{0}^{2 \pi} \int_{0}^{\pi} \int_{r_{i}}^{r_{o}} \rho c \theta(r, \psi, t) r^{2} d r \sin \psi d \psi d \varphi$

Hence, the heat flux has the form:

$Q=-2 \pi \rho c \sum_{n=0}^{\infty} \sum_{k=0}^{\infty} D_{k n} \int_{r_{i}}^{r_{o}} r^{2} \Phi_{n}\left(\omega_{k n} r\right) T_{k n}(t) d r$

By defining the dimensionless radius and time as,

$x=\frac{r}{r_{o}}, \bar{t}=\frac{t}{p}$

Eq. (21) can be rewritten in terms of Biot and Fourier numbers;

$B i=\frac{h r_{o}}{k}, F O=\frac{p}{a^{2} r_{o}^{2}}$

Since $f_{o}(\psi)$ is an arbitrary function, for example, we considered:

$f_{o}(\psi)=\sqrt{|\exp (|\cos (\psi)|)|}=\sqrt{|\exp (|\varsigma|)|}$

With the assumption of:

$\gamma_{k n}=\int_{r_{i}}^{r_{o}} r^{2} \eta_{n}(r) \Phi\left(\omega_{k n} r\right) d r$

and considering Eqs. (23) and (29), $T_{k n}(t)$ can be obtained:

$$
\begin{aligned}
& T_{k n}(\bar{t})=\gamma_{k n}\left(\frac{2 n+1}{2}\right) \int_{-1}^{+1} \sqrt{\exp (|\varsigma|) \mid} P_{n}(\varsigma) d \varsigma \\
& \int_{\tau=0}^{\bar{t}}\left(\bar{\Theta}+\sum_{m=0}^{\infty} \Theta_{M} \operatorname{Sin}(2 m \overline{\pi t})+\sum_{m=0}^{\infty} \bar{\Theta}_{M} \operatorname{Cos}(2 m \overline{\pi t})\right) e^{-\omega_{k n}^{2} F o(\bar{t}-\tau)} d \tau
\end{aligned}
$$

As $\bar{t} \rightarrow \infty$ all exponential terms should vanish, hence $T_{k n}(t)$ takes

the form:

$T_{k n}(\bar{t})=\frac{\gamma_{k n} a^{2}}{\omega_{k n}^{2}}\left(\frac{2 n+1}{2}\right) \int_{-1}^{+1} \sqrt{\exp (|\varsigma|) \mid} P_{n}(\varsigma) d \varsigma \times$

$\left[\bar{\Theta}+\frac{1}{\sqrt{1+\left(\frac{2 m M^{2}}{\omega_{k n}^{2}}\right)^{2}}}\left(\sum_{m=0}^{\infty} \Theta_{m} \operatorname{Sin}\left(2 m \pi \bar{t}+\phi_{k n}\right)+\sum_{m=0}^{\infty} \bar{\Theta}_{m} \operatorname{Cos}\left(2 m \pi \bar{t}+\phi_{k n}\right)\right)\right]$

where the dimensionless quantities $M$ and $\phi_{k n}$ are defined as:

$M=\sqrt{\frac{\pi a^{2}}{p}}$ 


$$
\phi_{k n}=\operatorname{Arctan}\left(-\frac{2 m M^{2}}{\omega_{k n}^{2}}\right)
$$

Thus, the temperature distribution in a hollow sphere for a periodic boundary condition when the inner boundary is insulated and the outer one dissipates heat into the ambient can be expressed in the form:

$\theta(\bar{r}, \psi, \bar{t})=\sum_{n=0}^{\infty} \sum_{k=0}^{\infty}\left(\frac{2 n+1}{2}\right) \int_{-1}^{+1} \sqrt{|\exp (|\varsigma|)|} P_{n}(\varsigma) d \varsigma\left(\frac{\Phi\left(\omega_{k n} \bar{r}\right) P_{n}(\varsigma) \gamma_{k n}}{\delta_{k n}}\right) \times$ $\left(\frac{\Theta_{m}}{\sqrt{1+\left(\frac{2 m M^{2}}{\omega_{k n}^{2}}\right)^{2}}} \times \sum_{m=0}^{\infty}\left(\operatorname{Sin}\left(2 m \pi \bar{t}+\phi_{k n}\right)+\operatorname{Cos}\left(2 m \bar{\pi}+\phi_{k n}\right)\right)\right.$

Substituting the eigenvalues into Eq. (34), the temperature distribution at the outer surface can be obtained in the form:

$$
\theta(1, \psi, \bar{t})=A_{m} \Theta_{m} \sum_{m=0}^{\infty}\left(\operatorname{Sin}\left(2 m \overline{\pi t}+\phi_{k n}\right)+\operatorname{Cos}\left(2 m \bar{t}+\phi_{k n}\right)\right)
$$

Where $A_{m}$ is the ratio of the oscillation amplitude of temperature distribution in the sphere and the ambient temperature with the

same frequency and $\phi_{k n}$ is the phase difference. By calculating and plotting Eq. (35), the maximum amplitude of summation of harmonic waves and the phase difference can be obtained.

\section{Results and Discussion}

To check our series solution, as a special case, we solved the problem of a solid sphere with a harmonic boundary condition with our method and compared the results with the corresponding ones in the literature [5]. As presented in Figs. 1 and 2, they are in excellent agreement.

It is evident from Figs. 1 and 2 that for small values of $M$ (slow oscillations of the outer ambient temperature), the dimensionless amplitude $A$ is almost 1 and the phase difference ) is approximately zero. For the large values of $M$ (fast oscillations of the outer ambient temperature), values of $A$ decrease from its maximum at the outer boundary to its minimum at the inner boundary and the phase difference values are negative. For the large enough values of $M$, only a thin boundary region of the sphere follows the ambient temperature oscillation. For increasing $\bar{r}_{i}$ at constant $\mathrm{Bi} / M$, the minimum of the phase difference decreases and occurs at larger $M$.

Figures 3-8 show the oscillation amplitude and the phase difference for the hollow sphere under a periodic boundary condition, which is decomposed using Fourier series with different radii and polar angles.

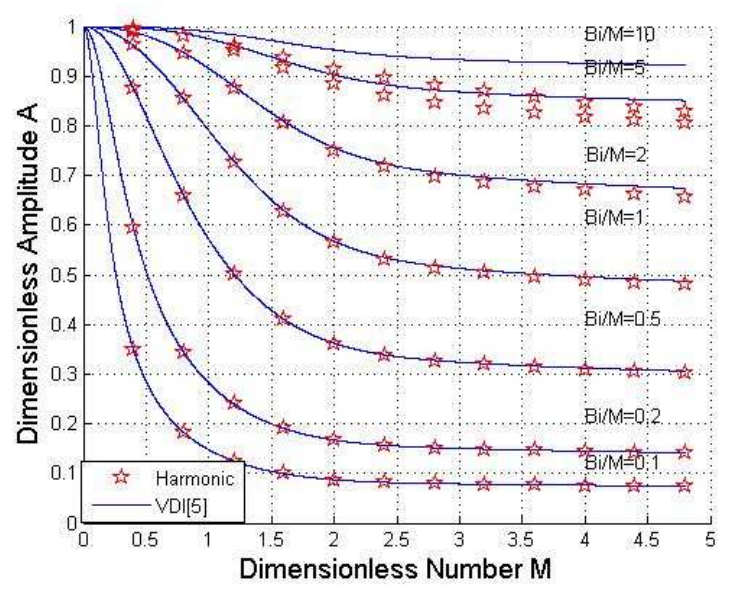

Fig. 1 Comparison between the result of the amplitude of a one-dimensional temperature field of a solid sphere [8] and the results for a solid sphere presented in [4] under the same boundary condition

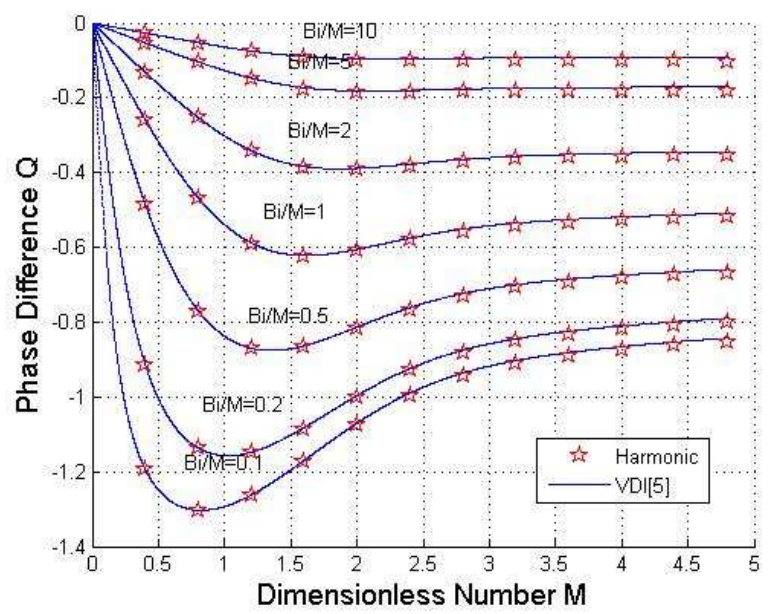

Fig. 2 Comparison between the result of the phase difference of a onedimensional temperature field of a solid sphere [8] and the results for a solid sphere presented in [4] under the same boundary condition

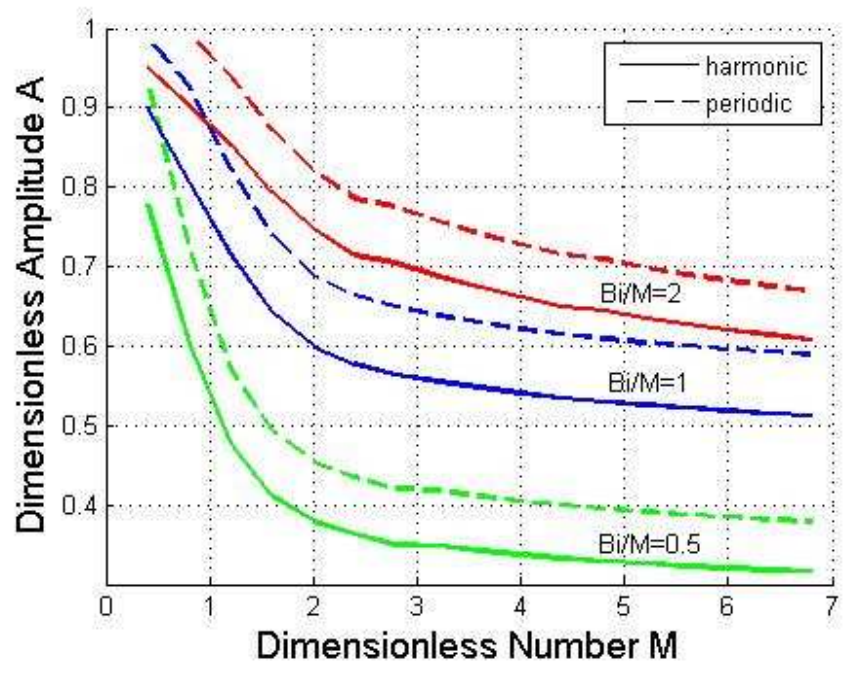

Fig. 3 Dimensionless amplitude, $A$, when $\bar{r}_{i}=0, \psi=0$ 


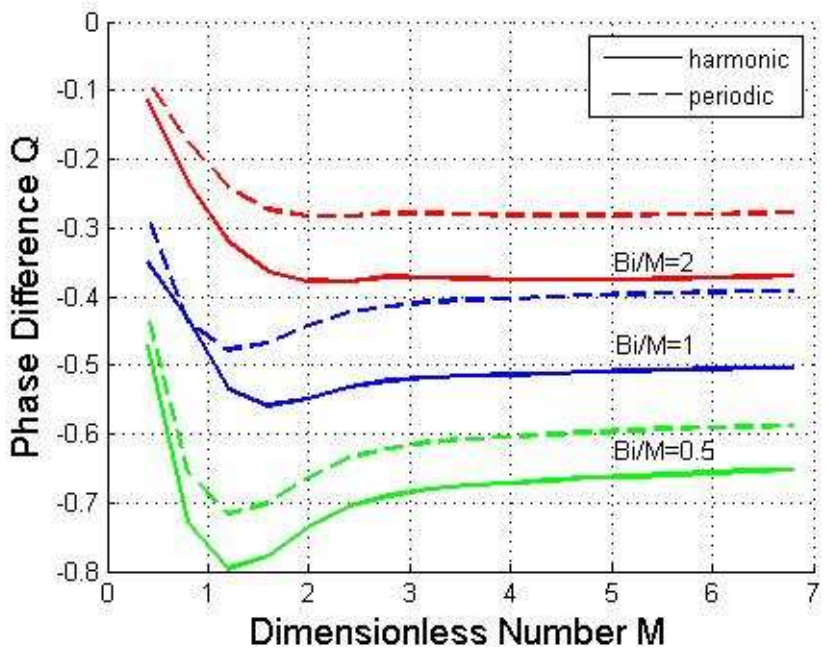

Fig. 4 Dimensionless phase difference, $\phi$, when $\bar{r}_{i}=0, \psi=0$

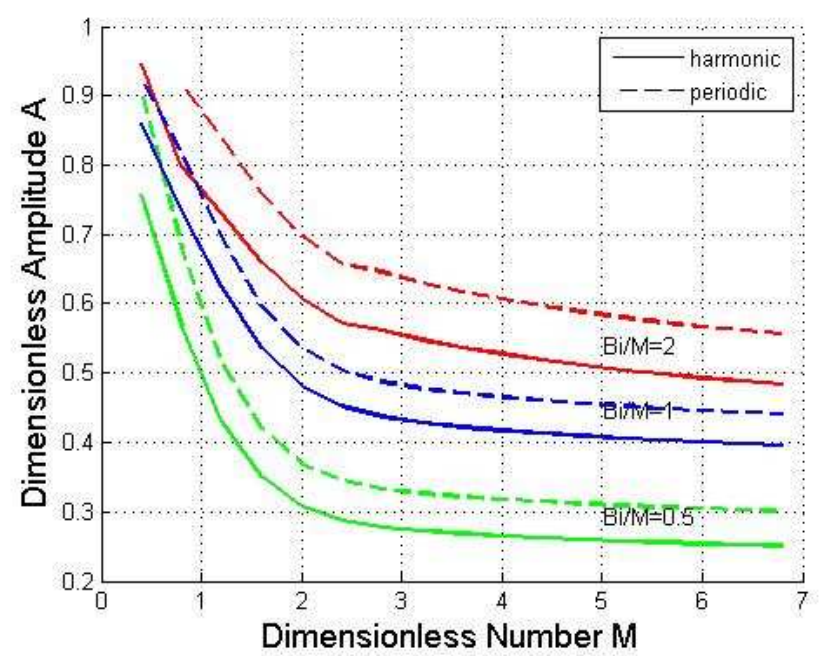

FIG. 5 DIMENSIONLESS AMPLITUDE, A, WHEN $\bar{r}_{i}=0, \psi=60$

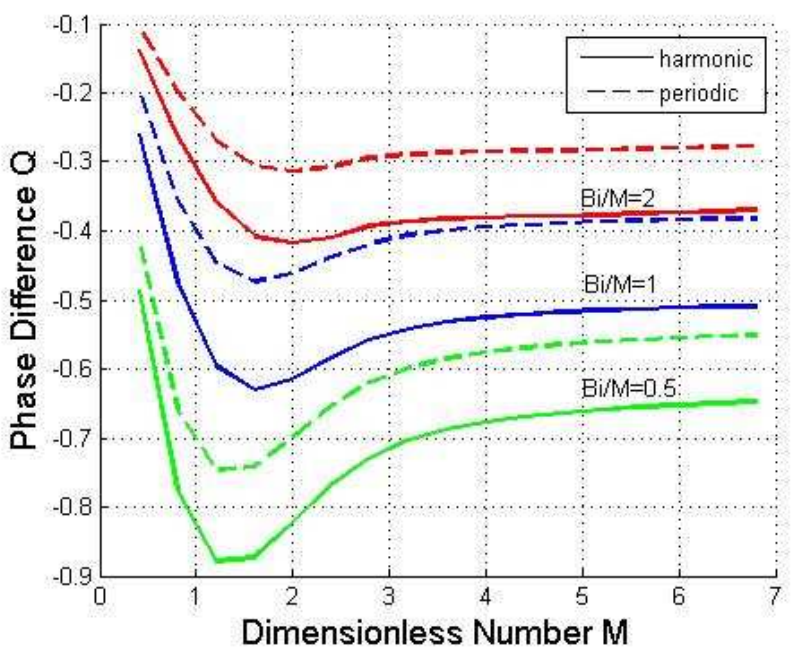

Fig. 6 Dimensionless phase difference, $\phi$, when $\bar{r}_{i}=0, \psi=60$

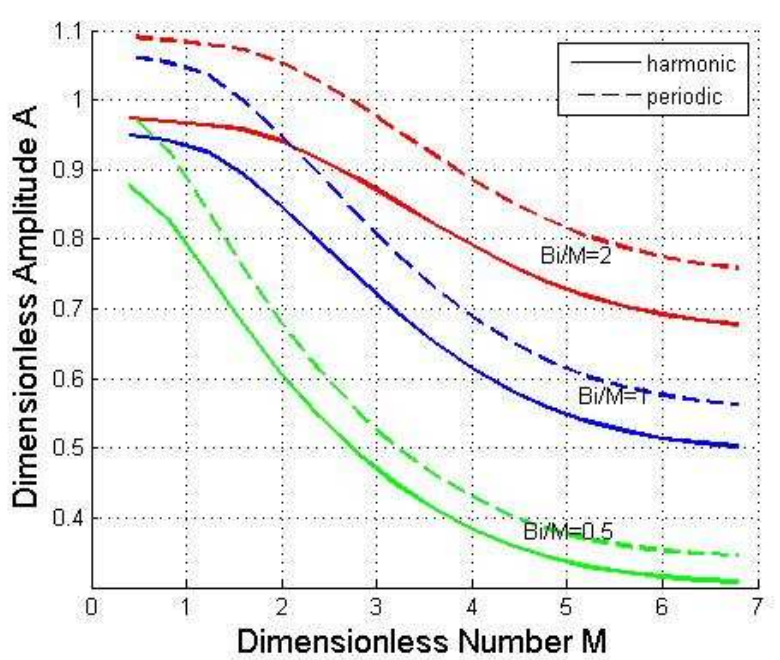

Fig. 7 Dimensionless amplitude, $A$, when $\bar{r}_{i}=0.8, \psi=0$

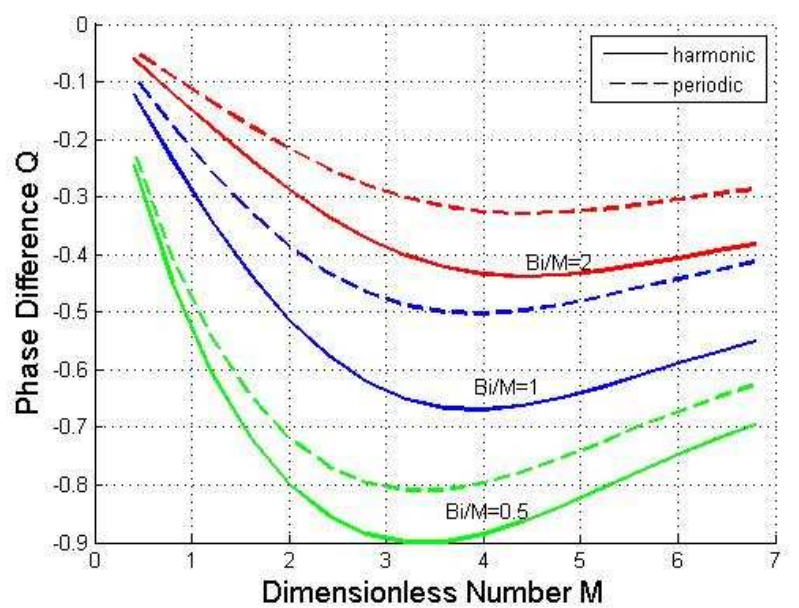

Fig. 8 Dimensionless phase difference, $\phi$, when $\bar{r}_{i}=0.8, \psi=0$

Figures 9 and 10 show the effect of $\bar{r}_{i}$ on the variations of $A$ and $\phi$ for the different values of $M$. For $\bar{r}_{i}<0.5, A$ and $\phi$ variations are not considerable but for $\bar{r}_{i}>0.5$, the effect of $\bar{r}_{i}$ is significant. When $\bar{r}_{i}$ tends to $1, A$ and $\phi$ tend to 1 and 0 , respectively. One can observe from Figs. 9 and 10 that with increasing $\bar{r}_{i}, A$ and $\phi$ do not increase necessarily, but for some values of $\bar{r}_{i}$ they decrease.

Figure 11 shows the effect of Biot number on the variation of $Q_{t} / Q_{p}$ for $\mathrm{M}=2, \quad \bar{r}_{i}=0.5$ under the periodic boundary condition. When the Biot number increases, the heat flux becomes larger and its value is positive in a half of a period and negative in another half. Figure 12 shows the effect of $\bar{r}_{i}$ on the variation of $Q_{t} / Q_{p}$ for $\mathrm{M}=2, \mathrm{Bi}=1$ under the periodic boundary condition. As Fig. 12 shows, when the thickness of the sphere decreases, the heat flux from the outer surface increases. 
Nomenclature

$a^{2}=$ inverse of thermal diffusivity, $\mathrm{s} / \mathrm{m} 2$

$c=$ specific heat capacity, $\mathrm{J} / \mathrm{kg} . \mathrm{K}$

$h=$ convection heat transfer coefficient, $\mathrm{W} / \mathrm{m} 2 . \mathrm{K}$

$k=$ thermal conductivity, $\mathrm{W} / \mathrm{m} . \mathrm{K}$

$p=$ period of the ambient temperature, $\mathrm{s}$

$r=$ radius, $\mathrm{m}$

$\mathrm{t}=$ Time, $\mathrm{s}$

$A=$ dimensionless amplitude

$\mathrm{Bi}=$ Biot number

$\mathrm{Fo}=$ Fourier number

$M=$ defined by Eq. (32)

$Q=$ heat flux, $\mathrm{W}$

$V=$ volume of the hollow sphere, $\mathrm{m} 3$

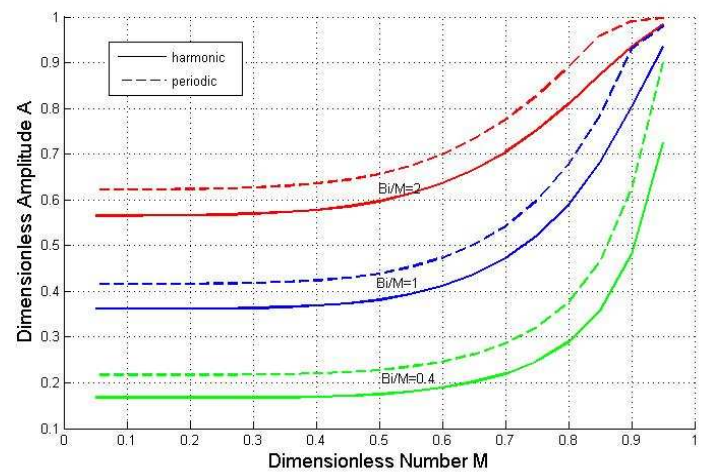

Fig. 9 Dimensionless amplitude, A, when $M=2, \psi=30$

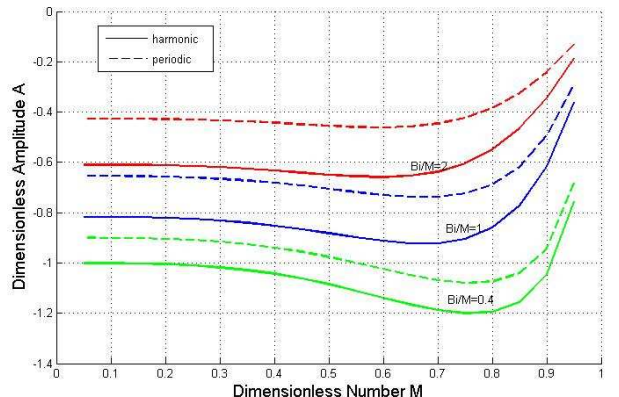

Fig. 10 Dimensionless phase difference, $\phi$, when $M=2, \psi=30$

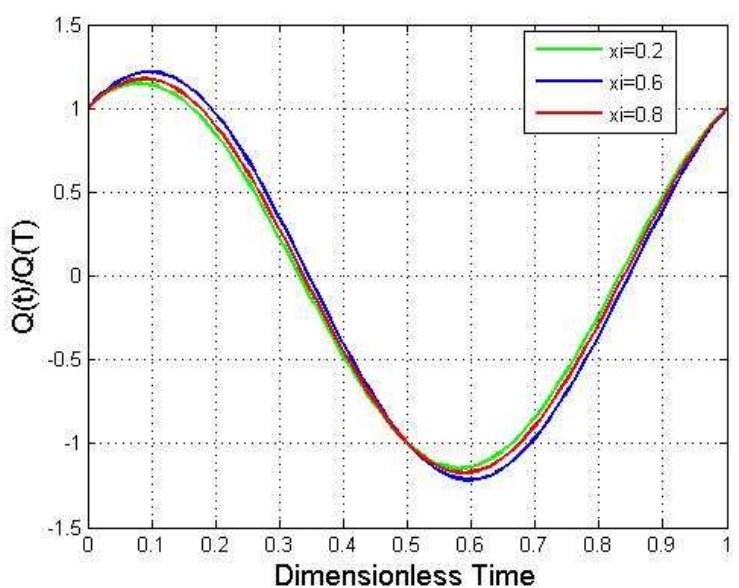

$\underline{Q(t)}$

FIG. $11 \overline{Q(p)}$ when $M=2, B i=1$ under periodic boundary Condition

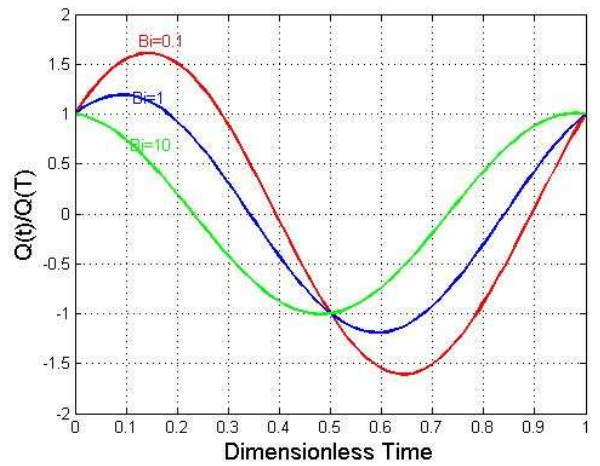

Fig. $12 \frac{Q(t)}{Q(p)}$ when $M=2$ under periodic boundary condition

\section{Greek letters}

$\theta=$ temperature, $\mathrm{K}$

$\omega=$ eigenvalue

$\phi=$ phase difference, $\mathrm{rad}$

$r, \phi, \psi=$ spherical coordinate

$\rho=$ density, $\mathrm{kg} / \mathrm{m} 3$

$\zeta=$ defined by Eq. (10)

$\eta=$ function defined by Eq. (12)

$\Phi=$ eigenfunction

\section{Subscripts}

$a=$ ambient

$i=$ inner

$o=$ outer

$s=$ steady state

$t=$ transient

\section{Superscript}

- $($ overbar $)=$ dimensionless quantity

\section{REFRENCES}

[1] Dincer, I., 1995, " Estimation of Dimensionless Temperature Distributions inSpherical Products During Hydrocooling," Int. Commun. Heat Mass Transfer, 22(1), pp. 123-131.

[2] Khedari, J., Benigni, P., Rogez, J., and Mathieu, J. C., 1995, "New Apparatus for Thermal Diffusivity of Refractory Solid Materials by the Periodic Stationary Method," Rev. Sci. Instrum., 66(1), pp. 193198.

[3] Khedari, J., Csurks, G., and Hirunlabh, J., 1998, "General Analytical Modeling of Heat Transfer in Isotropic Solid Materials Under Periodic Steady Regime," Proceedings of the International Conference on Contribution of Cognition to Modeling (CCM'98), Lyon-Villeurbanne, France, pp. 9.10-9.13.

[4] Verein Deutscher Ingenieure-Wärmeatlas. Hrsgeg vom Verein Deutscher Ingenieure, 2003, Ed 14-17, Düsseldorf.

[5] [5] Trostel, R., 1956, "Instationäre Wärmespannungen in einer Hohlkugel," Ing.- Arch., 24, pp. 373-391.

[6] Zubair, S. M., and Chaudhry, M. A., 1995, "Heat Conduction in Semiinfinite Solid Subject to Steady and Non-steady Periodic-Type Surface Heat Fluxes," Int. J. Heat Mass Transfer, 38(18), pp. 3393 3399.

[7] Özisik, M. N., 1993, Heat Conduction, 2nd ed., Wiley, New York, pp.195- 201.

[8] Atefi, G., and Shirmohammadi, R., 2003, "Analytical Solution of One- Dimensional Temperature fField in Solid Sphere Under Harmonic Boundary Condition," The 4th Iranian Aerospace Society Conference, Amirkabir University 